\title{
Synchronous EEG Brain-Actuated Wheelchair with Automated Navigation
}

\author{
I. Iturrate, J. Antelis and J. Minguez
}

\begin{abstract}
This paper describes a new non-invasive brainactuated wheelchair that relies on a P300 neurophysiological protocol and automated navigation. In operation, the subject faces a screen with a real-time virtual reconstruction of the scenario, and concentrates on the area of the space to reach. A visual stimulation process elicits the neurological phenomenon and the EEG signal processing detects the target area. This target area represents a location that is given to the autonomous navigation system, which drives the wheelchair to the desired place while avoiding collisions with the obstacles detected by the laser scanner. The accuracy of the brain-computer interface is above $94 \%$ and the flexibility of the sensor-based motion system allows for navigation in non-prepared and populated scenarios. The prototype has been validated with five healthy subjects in three experimental sessions: screening (an analysis of three different interfaces and its implications on the performance of the users), virtual environment driving (training and instruction of the users) and driving sessions with the wheelchair (driving tests along pre-established circuits). On the basis of the results, this paper reports a technical evaluation of the device and a variability study. All the users were able to successfully use the device with relative ease showing a great adaptation.
\end{abstract}

\section{INTRODUCTION}

Recently there has been a large impulse on the research and development of brain-controlled devices for rehabilitation. Following the brain-actuated robot control demonstrated in 2004 [1], there have been some attempts to develop a brain-actuated wheelchair. Some devices follow the clinical protocol where the EEG signals are synchronized with external cues, using one of the common event-related potentials (evoked potentials in the human brain associated with external stimuli, see [2] for review). For example, the wheelchair developed in Bremen [3] uses steady-state potentials and the Singapore one [4] uses the P300 potentials, both to select high-level primitives (e.g. go to the kitchen) in a menu-based system. Another synchronous device is the Espirito Santo University wheelchair, which uses the desynchronization of the alpha rhythms in the visual cortex that occurs when the eyes are open or closed [5] ${ }^{1}$. This desynchronization is used as a binary input to select low-level motion primitives (e.g. front, back, left, right) in a sweeping menu-based system. Another wheelchair concept was developed jointly in Leuven and IDIAP [6]. This device is based on an asynchronous protocol that analyzes ongoing EEG activity to determine the subject's mental state, which can change at any time. The

Iñaki Iturrate, Javier Mauricio Antelis and Javier Minguez are with the I3A and Dpto. de Informática e Ingeniería de Sistemas, Universidad de Zaragoza, Spain. E-mail: iturrate@unizar.es, antelis@unizar.es and jminguez@unizar.es

${ }^{1}$ Notice that this is a neurological phenomenon that requires the control of the blinking muscular process.

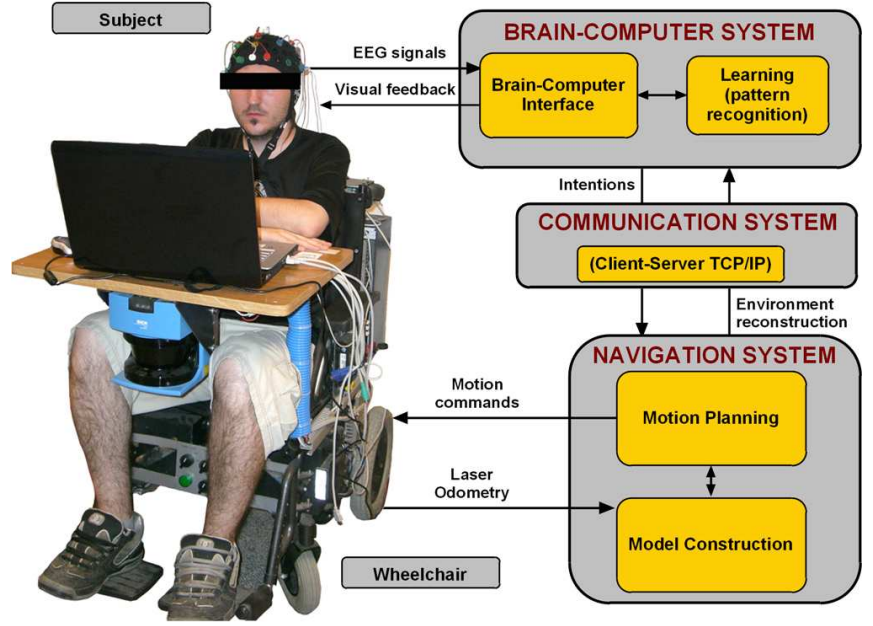

Fig. 1. This figure displays the mechatronic design of the brain-actuated wheelchair, the main modules and the information flow among them.

system deciphers the subject's steering directions (forward, right and left) and uses an obstacle avoidance system that executes the navigation.

This paper describes a new brain-actuated wheelchair concept that relies on a synchronous P300 brain-computer interface integrated with an autonomous navigation system (Figure 1). From the interaction point of view, this system has similar properties to the P300-based synchronous braincomputer interfaces (high accuracy but low transfer rate). From the navigation point of view, the advantage is that the user selects freely destinations of the environment (over the basis of a real-time reconstruction), which are safely and autonomously reached by the navigation system. This concept gives great flexibility to the user since the wheelchair can autonomously navigate in unknown and evolving scenarios using the onboard sensors. Furthermore, once the user has chosen the location he can relax, which avoids the exhausting mental processes of other devices.

We have validated the prototype with five healthy subjects in three steps: screening, virtual environment driving (training and instruction) and driving sessions with the wheelchair along pre-established circuits. On the basis of the results, this paper reports a technical evaluation of the device and of all the functionalities - i.e. the brain-computer interface and the navigation technology; and a variability study among trials and subjects. The overall result is that all the users were able to successfully use the device with relative ease showing a great adaptation, and also a high robustness and low variability of the system. 


\section{BRAIN-COMPUTER SYSTEM}

\section{A. Neurophysiological protocol and instrumentation}

The neurophysiological protocol followed in our study is based on an event-related response, the P300 visually evoked potential [7]. This potential manifests itself as a positive deflection in voltage at a latency of roughly $300 \mathrm{msec}$ in the EEG after the target stimulus is presented, within a random sequence of non-target stimuli (see Figure 2). A characteristic of this potential relevant to our BCI system is that neurophysiological studies [7] reveal that the elicitation time and the amplitude of the potential are correlated with the fatigue of the user and with the saliency of the stimulus (in terms of color, contrast, brightness, duration, etc).

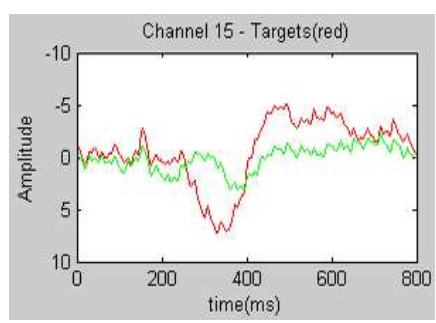

(a)

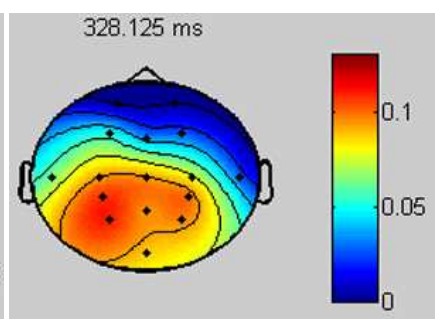

(b)
Fig. 2. (a) Typical P300 response. The red (dark) line shows the EEG activity on one channel (elicited by the target stimulus), and the green (light) line corresponds to the non-target one. (b) Topographical plot of the EEG distribution in the scalp at $300 \mathrm{msec}$. The area with more activity (mid-low part of the scalp) is in the parietal lobe, where the P300 potential is elicited.

The general instrumentation of the brain-computer interface $(\mathrm{BCI})$ is a commercial gTec EEG system (an EEG cap, 16 electrodes and a gUSBamp amplifier) connected via USB to the onboard computer. The location of the electrodes was selected according to previous P300 studies [8]. They were located at FP1, FP2, F3, F4, T7, T8, C3, C2, C4, $\mathrm{CP} 3, \mathrm{CP} 4, \mathrm{P} 3, \mathrm{P} 2, \mathrm{P} 4$ and $\mathrm{OZ}$ according to the international $10 / 20$ system. The ground electrode was positioned on the forehead (position Fz) and the reference electrode was placed on the left earlobe. The signal recording, processing and visual application was developed under the BCI2000 platform [9] and placed on a Intel Core2 Duo @ 2.10GHz running Windows XP OS. From now on, this computer will be mentioned as high-level computer.

The next subsection describes the graphical interface basis for the order selection and the visual stimulation process to elicit the P300 response in the user's EEG.

\section{B. Graphical interface}

In order to command the wheelchair, the user selects destinations or motion primitives by concentrating on the possibilities displayed on the computer screen (Figure 3a). The graphical interface displays information of the real-time reconstruction of the environment and additional information for the order selection; and develops the stimulation process to elicit the P300 visually-evoked potential. The graphical aspects of this module are based on a previous study involving a robotic wheelchair adapted for cerebral palsy users [10] with a tactile screen.

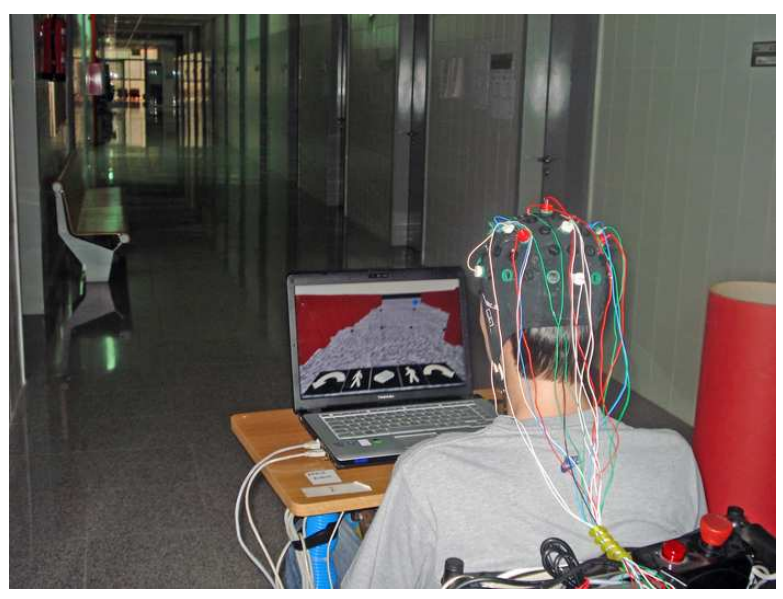

(a)

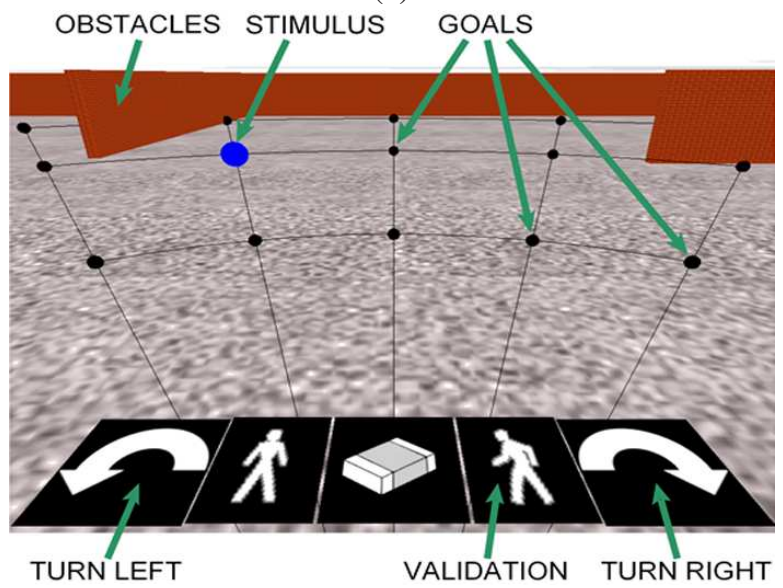

(b)

Fig. 3. (a) A snapshot that shows a subject navigating along a corridor (b) Representation of the visual display.

The information displayed on the screen is a reconstruction of the real scenario for the user's command selection (Figure $3 b)$. The environment $3 \mathrm{D}$ visualization is built from the $2 \mathrm{D}$ map constructed in real-time by the autonomous navigation technology (see subsection III). In other words, the visual information of the screen is a simplified reconstruction of the user's perception. The use of an online map instead of an a priori one endows the system with the flexibility to work in unknown scenarios. This is because online maps rapidly reflect changes in the environment, such as moving people or unpredictable obstacles like tables or chairs. The rest of the displayed information is used for command selection (Figure 3b). Firstly, there is a predefined set of destinations relative to the wheelchair location within the map, which corresponds to locations in the environment that the subject may select to reach. These places are represented in the display by an $N \times M$ polar grid attached to the wheelchair. The grid intersections represent real locations in the scenario, and its dimension is customizable. In this case, it was used a grid to represent locations at $(2 \mathrm{~m}, 4 \mathrm{~m}, 8 \mathrm{~m}) \times$ $\left(-60^{\circ},-30^{\circ}, 0^{\circ}, 30^{\circ}, 60^{\circ}\right)$ from the current wheelchair location, where the first grid row is the one that has the farthest destinations. The obstacles are depicted by walls, which hide 
the unreachable destinations of the grid. In addition to this, there are also specific actions available that are represented at the bottom of the visual display by icons. The arrow buttons turn the vehicle around $\pm 90^{\circ}$ its current position; the traffic light buttons $(i)$ validate the user's commands or (ii) stop the vehicle; and the rubber represents the "remove selection" option. In the current version of the interface the stop and "remove selection" options are not used, but they have been taken into account for the next interface prototype. All the elements of the visual display can be customized in terms of color, texture, shape, size and location. This was important in the screening sessions to equilibrate the user capabilities and preferences with the performance of the system (recall that the elicitation of P300 potential is affected by these issues).

The other aspect of the visual display is the stimulation process, used to elicit the P300 visual evoked potential when the user is paying attention to a given option. An option is "stimulated" by displaying a circle on the selection (Figure $3 b)$. One sequence of the stimulation process is a stimulation of all the options in a random order as required by the P300 oddball paradigm. In order to reduce the duration of a sequence and the dimension of the pattern recognition problem, we follow here the Farwell and Donchin [11] stimulation paradigm. In this paradigm, the flashing of the stimuli is done by means of rows and columns instead of flashing each option individually. Thus, in our interface there are 9 stimulations (rows plus columns) and two classification problems of 5 and 4 classes (the target option is the intersection of the target row and the target column). The number of sequences and all the scheduling of the stimulation process (time of exposition of each stimulus, inter-stimulus duration and inter-sequence duration) can be modified to equilibrate the user capabilities and preferences with the performance of the system.

\section{Pattern recognition strategy}

The pattern recognition is a supervised learning module that is trained to recognize the P300 evoked potential and thus to infer the stimulus that the user is attending to. The first step is to train the system via offline experiments, where the user faces the graphical interface with the stimuli described above. In this process, the user concentrates on a previously predefined sequence of selections that covers all the classes. The data is recorded and used to train the classification algorithm using a supervised learning technique of two steps described next.

1) Feature extraction: we follow [8] as feature extraction technique. The P300 signals are characterized in time domain so the information is in its waveform and latency times. In our study, for each EEG channel, samples in 1 second of recording were extracted after each stimulus onset. Then, these segments of data were filtered using the moving average technique and decimated by a factor of 16 . The resulting signals were plotted and the channels with the best P300 response were selected by visual inspection. The resulting data segments for each channel selected were concatenated, creating a single feature vector for the next stage.

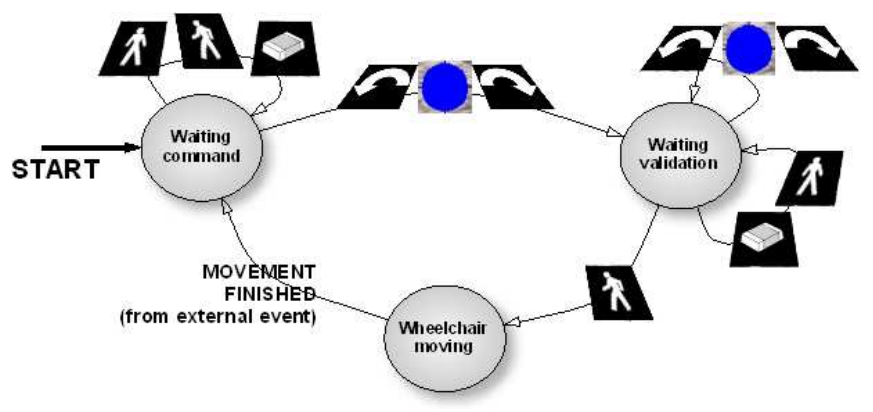

Fig. 4. Finite state machine that models the execution protocol of the options displayed on the screen so as to command the wheelchair.

2) Classification algorithm: the $P 300$ signal is elicited for one of the four rows or five columns during the sequence of stimulation. Thus, there are two classification problems of 4 and 5 classes. For each of these subproblems we used StepWise Linear Discriminant Analysis (SWLDA), extensively studied for P300 classification problems [8], and used with very good results in online communication using visual stimulation. In our system, with SWLDA we obtained a performance higher than $90 \%$ in less than an hour of training.

\section{Execution protocol}

The execution protocol is the way that the subject utilizes the possibilities of the visual display (Figure $3 b$ ). This protocol has been modeled by a finite state machine (Figure 4). Briefly, initially the state is Waiting command. In this state, the wheelchair is stopped, i.e without doing any action. Then, the user concentrates on one of the options, the BCI develops the stimulation process and, if there are no errors in the pattern recognition, the desired option is selected. When the option is a command (either a destination or a turn), the state turns to Waiting validation. In this state, the BCI develops the stimulation process again and a new option is selected. If the option is the validation, then the stimulation process is stopped, the relevant action is transferred to the autonomous system of the wheelchair (we refer to the pair command+validation as a mission) and the state turns to Wheelchair moving; otherwise, the stimulation process starts again until a new command is selected and later validated. Moreover, stop and "remove selection" options do not change the state nor the previous selection, which would remain selected. While the state is Wheelchair moving, the stimulation process is blocked, i.e there is no stimulation, waiting for an external flag coming from the autonomous navigation system (informing that the command has been executed). Once the flag is received the state turns to Waiting command.

\section{Robotic WhEELCHAIR}

The robotic wheelchair was constructed based on a commercial electric wheelchair that complied with basic user mobility and ergonomic requirements. We installed two Intel Pentium III $800 \mathrm{Mhz}$ computers on board. The first 


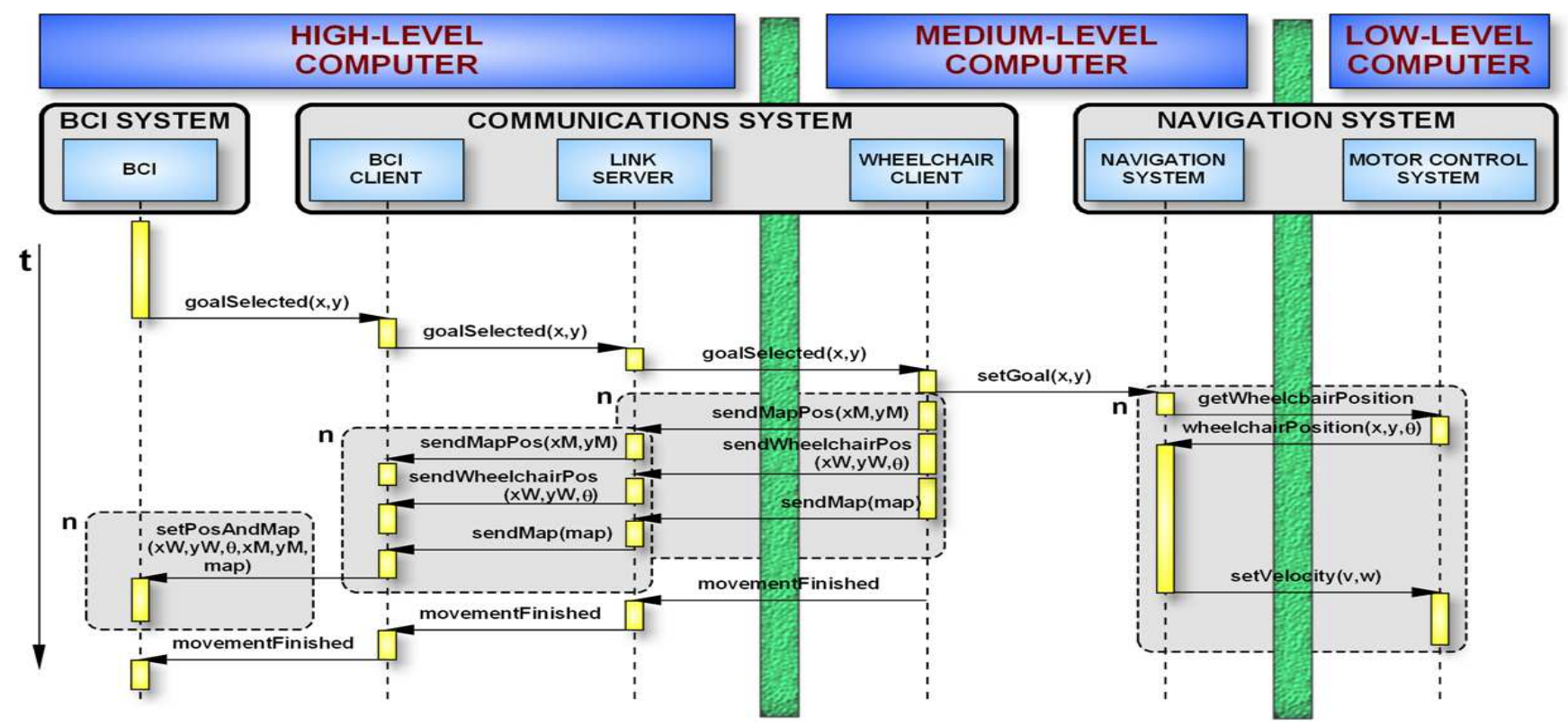

Fig. 5. The first row represents the computer hardware, whereas the second row represents the logical components. Below them, an event trace of the three computers integrated and running is shown, illustrating a typical flow of information starting when the user has selected a destination. The flow of information and its direction are illustrated by arrows. Vertically, time increases downward, and the vertical rectangles below boxes stand for an execution of code. The dark boxes enveloping certain portions of code and information exchange represent an iterative execution task.

computer performs the low-level control (real-time operative system, VxWorks) controlling the back wheels that work in differential-drive mode. The second computer is used for medium-level control, performing the navigation computations and managing the communications between the wheelchair and the BCI system. The main sensor is a SICK planar laser placed in the frontal part of the vehicle. It works at $5 \mathrm{~Hz}$, with a field of view of $180^{\circ}$ and $0.5^{\circ}$ resolution (361 points). This sensor provides information about the obstacles in front of the vehicle. The wheelchair is also equipped with wheel encoders to measure the odometry (position and orientation).

We have incorporated to the robot an autonomous navigation technology that is able to drive the vehicle to a given destination while also avoiding the obstacles, both static and dynamic, detected by the laser sensor [12]. This module has two functionalities. On the one hand, a modeling module integrates the sensor measurements to construct a local model of the environment and track the vehicle location. We chose a binary occupancy grid map to model the static obstacles and the free space, and a set of extended Kalman filters to track the moving objects around the robot. We use a given technique [13] to correct the robot position, update the map and detect and track the moving objects around the robot . The static map travels centered in the robot. This map has a limited size, which is enough to present the required information to the user as described in the previous section and to compute the path so as to reach the selected goal. On the other hand, a local planner computes the local motion based on the hybrid combination of tactical planning and reactive collision avoidance. We use an efficient dynamic navigation function $\left(\mathrm{D}^{*}\right.$ Lite planner [14]) to compute the tactical information (i.e. main direction of motion) required to avoid cyclic motions and trap situations. This function is well suited for unknown and dynamic scenarios because it works based on the changes in the model computed by the model builder. The final motion of the vehicle is computed using the ND technique [15], which uses a "divide and conquer" strategy based on situations and actions to simplify the collision avoidance problem. This technique has the distinct advantage that it is able to cater to the complex navigational tasks such as maneuvering in the environment within constrained spaces (e.g., passage through a narrow door). In order to facilitate comfortability and safe operation during navigation, we incorporated the shape, kinematics and dynamic constraints of the vehicle [12].

\section{COMmunication System AND InTEgRATION}

The interaction between the brain-computer interface and the autonomous navigation system is carried out by the communication system (Figure 1). This system is based on a TCP/IP connection between the high-level computer and the medium-level computer of the wheelchair (Figure 5). The software architecture is composed by a server and two clients integrated within the previous systems: $(i)$ the BCI client is multiplexed in time with the BCI system with a period of 30 msec; (ii) the wheelchair client encapsulates the navigation system as a thread, and (iii) a link server, located between the clients, concentrates the information flow and makes the system scalable for further additions.

The temporal information flow and synchronization of the modules are displayed in Figure 5. A typical execution is: first the BCI computes a goal location ( 8 bytes of information) which is transferred to the link server via the BCI client. 


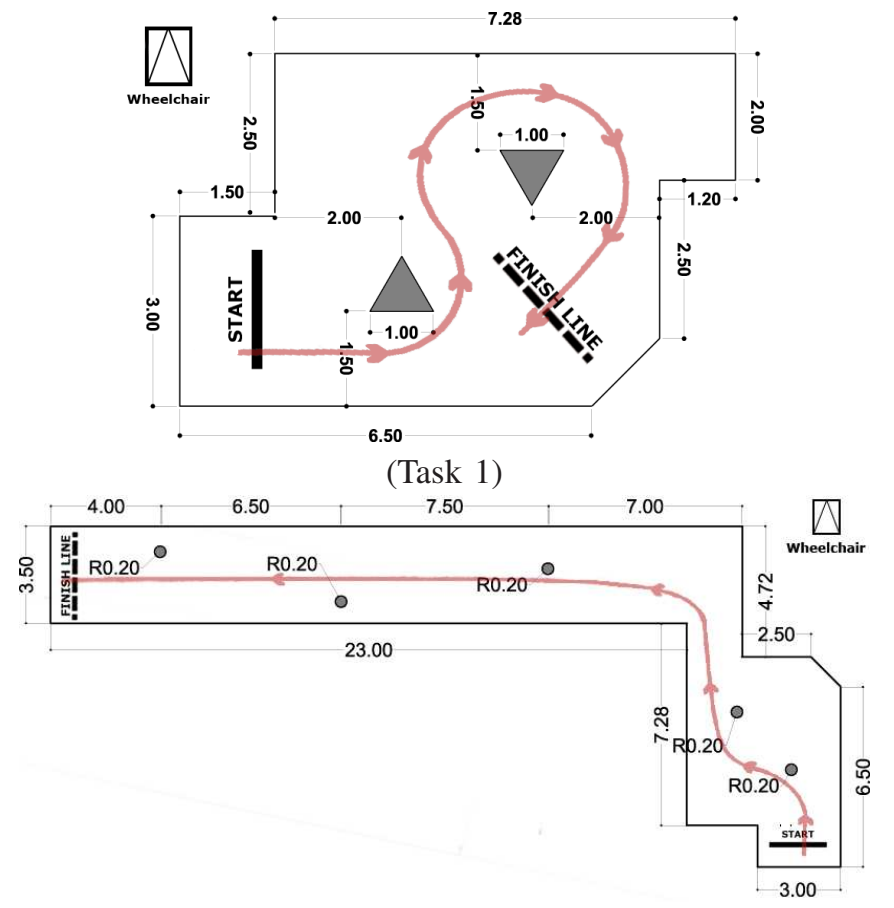

(Task 2)

Fig. 6. The objective of Task 1 was to leave the start area and to reach the finish line by leaving the first triangle on the left-hand side, passing between the two triangles and leaving the last triangle on the right-hand side. The objective of Task 2 was to reach the finish line from the start. For the shake of simplicity, a path has been drawn in red in both figures to give an overall idea to the reader. All measures are in meters and the wheelchair is to scale. The shaded objects represent static obstacles.

The client of the navigation system gets this information from the server and makes it available for the navigation system. Within a synchronous periodical task of $0.2 \mathrm{sec}$, the navigation system reads the goal location from the motor control system and the laser sensor, requests the robot odometry from the low-level computer, executes the mapping and planning module and sends the computed translational and rotational velocity to the low-level computer. There are three variables computed by the navigation system that need to be transferred to the BCI (the map model of 400 bytes, the model location of 12 bytes and the wheelchair location within the map of 12 bytes). These variables, located in the navigation thread are accessible in mutual exclusion by its client, which sends them to the link server that transfers them to the BCI client. When the wheelchair reaches the final location, the navigation system triggers and sends a flag to stop this information transfer process. Then, the BCI starts again the stimulation process to get a new goal location.

The maximum bandwidth between the high-level computer and the medium-level computer of the wheelchair is 2 kbytes/sec (when the navigation system is moving the wheelchair). The bandwidth of the communication between the computers of the wheelchair is $0.1 \mathrm{kbytes} / \mathrm{sec}$. Both information transfer rates do not overwhelm the internal network and do not impose a significant computation time for the clients and servers.

\section{Experimental Methodology}

The objective of this study was to assess the performance and adaptability of the brain-controlled mobility device (wheelchair) driven by able-bodied users in real settings. In the following sections, we discuss the recruitment of the participants for the study followed by a summary of the experimental protocol.

\section{A. Participants}

The recruitment for participation in the study began after obtaining the approval of the protocol by the University of Zaragoza Institutional Review Board. The selection for the participation was made by the research team.

A set of inclusion and exclusion criteria were applied for the recruitment of users in order to obtain the conclusions for the study over a homogeneous population. The inclusion criteria were: $(i)$ 20-25 years old; (ii) sex (either all women or all men); (iii) laterality (either all left-handed or all right-handed); and (iv) students of the engineering school of the University of Zaragoza. The exclusion criteria were: $(i)$ subjects with history of neurological or psychiatric disorders; (ii) subjects under any psychiatric medication; and (iii) subjects with episodes of epilepsy, dyslexia or experiencing hallucination. Finally, in addition to these criteria, we were constrained with the ergonomic conditions so as to suit the users to the wheelchair size and design: $(i)$ the subject weight had to be $60 \pm 20 \mathrm{~kg}$; (ii) the height had to be $1.70 \pm 0.20 \mathrm{~m}$; and (iii) the body posture had to be medium or thin.

Five healthy, 22 years old, male and right-handed students of the University participated in the experiments. None of them had ever utilized an electric wheelchair before. The participants were duly informed about the whole protocol of the study before they signed the consent forms. Permission to reproduce video recording and photographic images were duly taken from the subjects.

\section{B. Experiment Design and Procedures}

The study was accomplished in three phases in the BCI laboratory of the University of Zaragoza. Each phase lasted one week. We summarize next the first two phases, and focus on the third phase since it involves the rehabilitation device.

The first phase was the screening session. The objective of this session was to screen the subjects for the next stage and to come up with a graphical interface that equilibrates the user capabilities and preferences with the performance of the system, in terms of color and brightness of the stimulus and environment textures. To bound the complexity of the experiment, we designed three different groups of aesthetic factor of the interface (explained in subsection II-B), and performed a screening for each of them. For each subject, this session lasted for 3 hours. The second phase consisted of a training subphase (subsection II-C) and on a test in a wheelchair simulator, which emulates the underlying mechanisms of the user interface and the wheelchair navigation. After passing the training phase, they participated in this driving test that consisted of a navigation trial with the wheelchair simulator 

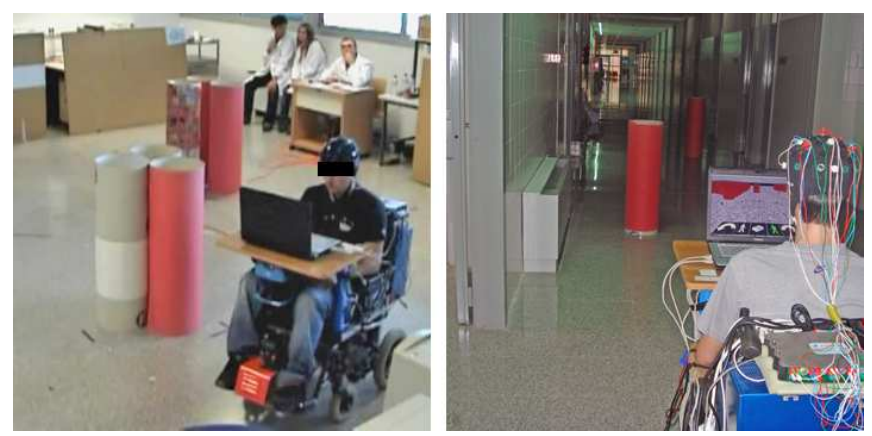

Fig. 7. Snapshots of different subjects during the experiments in the "S" circuit and in the "corridor" circuit.

along a circuit common for all subjects. For each subject, this session lasted for 3 hours.

The last phase consisted of real-time navigation with the wheelchair along pre-established circuits. The objective of this battery of experiments was to create the basis for a technical and variability evaluation of the brain-actuated wheelchair: to explore the navigation capabilities of the system and to assess the performance of the subjects in real settings. We designed two circuits that the user had to solve by autonomously navigating with the wheelchair (Figure 6). The first circuit was designed to accomplish complex maneuverability tasks and avoidance of obstacles in constrained spaces. The second circuit involved a navigation in open spaces. Each subject performed two trials of the first circuit (named "S" circuit) and then other two trials for the second circuit (named "corridor" circuit). For each subject, this session lasted for 4 hours.

\section{Results AND Evaluation}

This section reports the results of the experiments previously described. Notice that the experimental methodology has two preparatory phases before the usage phase of the rehabilitation device. For space reasons, we briefly outline the results of these preparatory phases (the reader is directed to [16] for more details about this evaluation) to focus on the evaluation of the brain-actuated device itself.

After the first experimentation phase (screening session) there was an evaluation process to select the group of factors that better equilibrated user capabilities and preferences with the performance of the system. We established various technical metrics and psychological metrics, obtaining the best result with the group of factors showed in Figure $3 \mathrm{~b}$. Once second phase (driving test) was executed, there was a study of each subject's navigation. The result was that all the subjects passed the test, since they showed an acceptable understanding of the interface and navigation performance. Thus, they all qualified for the real wheelchair navigation.

The last phase consisted of real-time navigation on the wheelchair along the pre-established circuits. The overall result of the experiments is that all the subjects were able to carry out the navigation tasks along the circuits with relative ease (Figure 7). On the basis of these experiments, in this section we describe an evaluation of the rehabilitation device. We focus on two different points of view: a performance study of the intelligent wheelchair and a variability study among trials and subjects.

\section{A. Intelligent Wheelchair Performance Evaluation}

This subsection describes a general evaluation of the brain-actuated wheelchair and a particular evaluation of its two main systems: the brain-computer interface and the navigation technology.

1) Overall performance: We follow here the metrics proposed in [10] to evaluate the performance of autonomous wheelchairs:

- Task success: degree of accomplishment of the task.

- Path length: distance traveled to accomplish the task.

- Time: time taken to accomplish the task.

- Collisions: number of collisions.

- BCI accuracy: accuracy of the pattern recognition.

- Number of missions: the global navigation task is accomplished by iteratively setting navigation missions.

The results (on average of every subject) are summarized in table I.

TABLE I

MEtrics to EVALUATE THE WHEELCHAIR PERFORMANCE

\begin{tabular}{l|cccc|cccc} 
& \multicolumn{5}{|c|}{ S-Path circuit } & \multicolumn{4}{c}{ Corridor circuit } \\
& $\min$ & $\max$ & mean & std & $\min$ & $\max$ & mean & std \\
\hline Path length (m) & 12.8 & 19.0 & 15.7 & 2.0 & 37.5 & 41.4 & 39.3 & 1.3 \\
Time (sec) & 448 & 834 & 571 & 123 & 507 & 918 & 659 & 130 \\
Useful BCI acc. & 0.88 & 1 & 0.95 & 0.04 & 0.81 & 1 & 0.94 & 0.07 \\
\# of missions & 8 & 14 & 9.6 & 1.9 & 7 & 12 & 9.2 & 2.9
\end{tabular}

All the subjects succeeded to autonomously navigate along the two circuits, which is the best indicator of the device utility. No collisions occurred during the experiments because of the autonomous navigation system. The path length, time taken and number of missions was very similar for all the subjects indicating a similar performance across subjects. From the BCI point of view, the interaction with the wheelchair was also satisfactory since the performance of the pattern recognition system wwas above $94 \%$ on average. We understand that all these results are very encouraging since the experiments were carried out in scenarios carefully designed to cover many of the typical real navigation situations of these devices.

2) Brain-Computer System: We divide this evaluation in an evaluation of the pattern recognition and another one of the design of the graphical interface. There have been some metrics proposed to evaluate pattern recognition strategies [17]. Based on them, we propose the following measures:

- Real BCI accuracy: BCI correct selections vs total.

- Total errors: number of incorrect selections.

- Useful errors: incorrect selections of the BCI that the user decided to reuse.

- Useful BCI accuracy: correct selections plus useful errors vs total.

The results are summarized in table II. 
TABLE II

METRICS TO EVALUATE THE PATTERN RECOGNITION

\begin{tabular}{l|cccc|cccc} 
& \multicolumn{4}{|c|}{ S-Path circuit } & \multicolumn{4}{c}{ Corridor circuit } \\
& min & $\max$ & mean & std & $\min$ & $\max$ & mean & std \\
\hline Real BCI acc. & 0.85 & 1 & 0.93 & 0.05 & 0.77 & 1 & 0.92 & 0.07 \\
Useful BCI acc. & 0.88 & 1 & 0.95 & 0.04 & 0.81 & 1 & 0.94 & 0.07 \\
\# Total errors & 0 & 4 & 1.6 & 1.35 & 0 & 7 & 1.9 & 2.13 \\
\# Useful errors & 0 & 1 & 0.3 & 0.48 & 0 & 1 & 0.4 & 0.52
\end{tabular}

The real accuracy on average was greater than $92 \%$, indicating a high accuracy. We have distinguished between real and useful accuracy. This is because in some situations, although the BCI system did not recognize the user's selection, the BCI selection was used by the subject to achieve the mission. These useful errors was almost $20 \%$ of the total errors making the useful accuracy greater than the real one. Furthermore, they reduced the number and the time for selections and validations. Notice that, in general, the effect of a BCI error was only a delay in the execution time until a new selection was done. During the experiments, the BCI system never set an incorrect mission to the autonomous navigation system. This is because the probability of this situation is below $0.3 \%$ (in the usage protocol there must be first a BCI failure in a selection and second another BCI failure that results in selecting the validation option).

Regarding the graphical interface, we adapted the metrics proposed in [10]: usability rate (i.e. number of selections per mission), command utility (i.e. command usage frequency) and the number of errors by misunderstanding of the interface. The results are summarized in table III.

TABLE III

\begin{tabular}{|c|c|c|c|c|c|c|c|c|}
\hline \multicolumn{9}{|c|}{ METRICS TO EVALUATE THE GRAPHICAL INTERFACE } \\
\hline & $\min$ & $\begin{array}{l}\text { S-Pa } \\
\max \end{array}$ & $\begin{array}{c}\text { circuit } \\
\text { mean }\end{array}$ & std & $\min$ & $\begin{array}{c}\text { Corric } \\
\max \end{array}$ & $\begin{array}{l}\mathrm{r} \text { circuit } \\
\text { mean }\end{array}$ & std \\
\hline \# 1st grid row & 0 & 1 & 0.1 & 0.3 & 0 & 4 & 2.7 & 1.3 \\
\hline \# 2nd grid row & 0 & 4 & 1.7 & 1.1 & 1 & 10 & 4.3 & 2.8 \\
\hline \# 3rd grid row & 1 & 9 & 5.6 & 2.4 & 1 & 9 & 3.7 & 2.5 \\
\hline \# Turns & 0 & 6 & 1.6 & 1.27 & 0 & 1 & 0.1 & 0.31 \\
\hline \# of validations & 8 & 14 & 9.6 & 1.9 & 7 & 12 & 9.2 & 1.9 \\
\hline \# Usability rate & 2.0 & 2.4 & 2.1 & 0.1 & 2.0 & 2.6 & 2.2 & 0.2 \\
\hline \# Misunderstandings & 0 & 0 & 0 & 0 & 0 & 0 & 0 & 0 \\
\hline
\end{tabular}

In general, the design of the interface was acceptable, since all the subjects were able to use it to carry out the navigation task. The command utility was greater than zero for all the subjects and commands, indicating that they used all the functionalities of the screen. The frequency of usage of some commands (far goals and turns) suggests that the users perform the driving tasks in a different way, as reported in similar studies [10]. Regarding the usability rate, the mean of the rate indicates very low extra selection rate (in theory it is needed 2 selections/mission). Notice that this increase could come from BCI errors (see above) and from misunderstandings of the interface (which are the ones that affect the interface design). Although there were no errors of misunderstanding reported by the subjects, we cannot eliminate the possibility that errors occurred but subjects did not become aware of them.

Regarding the $\mathrm{BCI}$ in general, an important aspect is the information transfer rate (i.e. number of bits per minute trans- ferred from the user to the machine). The stimulation process took always around 25 seconds (more than 2 selections per minute). Thus, the information transfer rate was 12 bits per minute, since the recognition time is below one second.

In summary, these results indicate that the pattern recognition strategy and the graphical interface of the braincomputer interface were suitable for controlling the intelligent wheelchair.

3) Navigation system performance: To evaluate the navigation of the robot, we propose as metrics [10]: the number of successful missions, the number of collisions per mission and per time and the obstacle clearance (i.e. minimum and mean distance to the obstacles). The results are summarized in table IV.

TABLE IV

\begin{tabular}{l|cccc|cccc}
\multicolumn{8}{c}{ METRICS TO EVALUATE THE NAVIGATION SYSTEM } \\
& \multicolumn{7}{c}{ S-Path circuit } & \multicolumn{5}{c}{ Corridor circuit } \\
& min & max & mean & std & min & max & mean & std \\
\hline \# of missions & 8 & 14 & 9.60 & 1.90 & 7 & 12 & 9.20 & 1.93 \\
Path length (m) & 12.84 & 19.02 & 15.74 & 1.99 & 37.52 & 41.44 & 39.31 & 1.33 \\
Velocity (m/sec) & 0.10 & 0.15 & 0.13 & 0.01 & 0.16 & 0.19 & 0.18 & 0.01 \\
Time in motion (sec) & 100 & 160 & 124.4 & 19 & 206 & 247 & 220 & 12 \\
Clearance min (m) & 0.67 & 0.88 & 0.79 & 0.07 & 0.47 & 0.71 & 0.61 & 0.07 \\
Clearance mean (m) & 2.83 & 3.16 & 3.02 & 0.12 & 3.19 & 3.34 & 3.28 & 0.05
\end{tabular}

The performance of the navigation system was remarkable since all the missions were successfully solved. In total, the system carried out 188 missions traveling a total of 550.5 meters with on average velocity of $0.16 \frac{\mathrm{m}}{\mathrm{sec}}$ ( 5 times less than the usual human walking velocity). There were no collisions during the experimentation. One of the main difficulties of current navigation systems is to avoid the obstacles with safety margins and to drive the vehicle between close obstacles [12]. The mean of the minimum clearance was 0.79 and 0.61 and the clearance mean was 3 and 3.28 , which means that the vehicle carried out obstacle avoidance with safety margins.

In general the navigation system successfully solved all the navigation missions without collisions in environments with different conditions and constraints.

\section{B. Variability Analysis}

This study analyzes two types of variability degrees during the experimental sessions: (i) intra-subject variability measures the variability of a subject among trials of the same task, and (ii) inter-subject variability measures the variability of execution among subjects during the execution of the same task. Within these results, the aim of this analysis is also to infer the degree of homogeneity of the system developed, i.e whether a homogeneous group of participants offers similar results in similar experimental conditions. We propose the number of selections, missions, distance and useless BCI failures normalized in time as the basis for this variability study. To measure the variability, we apply to these metrics Pearson's correlation coefficient: values close to one indicate low variability, while values far from one indicate high variability.

The intra-subject variability was greater than 0.94 (except for Subject 2 in Task 1) indicating that the variability among 
trials was not substantial. This low intra-variability denotes the users determined that his way to resolve the task was correct, and therefore they tried to perform equally in both executions.

As far as inter-subject variability is concerned, results of this analysis are shown in table $\mathrm{V}$.

\section{TABLE V}

\begin{tabular}{|c|c|c|c|c|c|c|c|c|c|c|}
\hline \multicolumn{11}{|c|}{ INTER-SUBJECT VARIABILITY } \\
\hline & \multicolumn{5}{|c|}{ Task 1} & \multicolumn{5}{|c|}{ Task 2} \\
\hline & S1 & S2 & S3 & S4 & S5 & S1 & S2 & S3 & S4 & S5 \\
\hline S1 & 1 & 0.962 & 0.987 & 0.953 & 0.981 & 1 & 0.960 & 0.917 & 0.953 & 0.998 \\
\hline S2 & - & 1 & 0.944 & 0.952 & 0.977 & - & 1 & 0.964 & 0.988 & 0.970 \\
\hline S3 & - & - & 1 & 0.978 & 0.980 & - & - & 1 & 0.990 & 0.926 \\
\hline S4 & - & - & - & 1 & 0.984 & - & - & - & 1 & 0.961 \\
\hline S5 & - & - & - & - & 1 & - & - & - & - & 1 \\
\hline
\end{tabular}

The coefficient was greater than 0.92, indicating a low inter-variability. This low variability denotes that the users executed the task in a similar and analogous way. These results, together with those of intra-variability allows to infer that in the same experimental conditions, the group performs similar actions, giving to the system a high degree of homogeneity and invariability against these situations.

\section{CONCLUSIONS}

This paper describes a new brain-actuated wheelchair concept that relies on a synchronous P300 brain-computer interface integrated with an autonomous navigation system. This combination gives great accuracy in the interaction and flexibility to the user, since the wheelchair can autonomously navigate in unknown and evolving scenarios using the onboard sensors.

The system was used and validated by five healthy subjects in three consecutive steps: screening, virtual environment driving and wheelchair driving sessions. During the real experiments, the system showed high performance since all the subjects accomplished two different tasks with relative easiness. The experiments were carried out in settings designed to cover typical navigation situations, such as open spaces and complex maneuverability. The BCI accuracy, the performance of the graphical interface and the performance of the navigation system was high, indicating that the integration of these technologies was satisfactory. Furthermore, the variability study suggests that the results have a low variability, giving the system a high degree homogeneity.

In the near future, we are working on the improvement of the system to address the common problem of all eventrelated potential approaches: the low information transfer rate. In this direction, we are developing a P300 continuous control of the system, in an attempt to reduce the recognition time. Another shortcoming of these systems is that with the synchronous operation, the user has to be continuously concentrated in the task. An interesting improvement the researchers would like to work is the adoption of asynchronous P300 control to support an idle state. Although the BCI accuracy is high (94\%), we are also working on the integration of BCI-based online error detection system (which is a direction followed in many laboratories).
As future work, it would also be important to perform experimental validation with potential users of the system developed. These users are those who have lost almost all voluntary muscle control, because of diseases such as Amiotrophic Lateral Sclerosis (ALS), spinal cord injury or muscular dystrophies for example.

\section{ACKNOWLEDGEMENTS}

We thank Sonu Bhaskar, Carlos Escolano, María López, Belén Masiá and all the participants in the experiments for their support in the development of the project. This work has been partly supported by the Spanish project DPI2006-15630-C02-02.

\section{REFERENCES}

[1] J.d.R. Millán, F. Renkens, J. Mouriño, and W. Gerstner, "Noninvasive Brain-Actuated Control of a Mobile Robot by Human EEG," IEEE Transactions on Biomedical Engineering, vol. 51, no. 6, June 2004.

[2] J.R.Wolpaw, N.Birbaumer, D.J.McFarland, G.Pfurtscheller, and T.M.Vaughan, "Brain-computer interfaces for communication and control," Clinical Neurophysiology, vol. 113, no. 6, pp. 767-91, 2002.

[3] T. Luth, D. Ojdanic, O. Friman, O. Prenzel, and A.Graser, "Low level control in a semi-autonomous rehabilitation robotic system via a Brain-Computer Interface," 2007.

[4] B. Rebsamen, C.L. Teo, Q. Zeng, M.H. Ang, E. Burdet, C. Guan, H. Zhang, and C. Laugier, "Controlling a Wheelchair Indoors Using Thought," IEEE Intelligent Systems, vol. 07, pp. 1541-1672, 2007.

[5] A. Ferreira, R.L. Silva, W.C. Celeste, T.F. Bastos, and M. Sarcinelli, "Human-Machine Interface Based on Muscular and Brain Signals applied to a Robotic Wheelchair," IOP Publishing Ltd., vol. 07, pp. 1541-1672, 2007.

[6] G. Vanacker, J.d.R.Millán, E. Lew, P. W.Ferrez, F.G. Moles, J. Philips, H. V. Brussel, and M. Nuttin, "Context-Based Filtering for Assisted Brain-Actuated Wheelchair Driving," Computational Intelligence and Neuroscience, May 2007.

[7] S.H. Patel and P.N. Azzam, "Characterization of N200 and P300: Selected Studies of the Event-Related Potential," International Journal of Medical Sciences, pp. 147-154, October 2005.

[8] D. J. Krusienski, E. W. Sellers, F. Cabestaing, S. Bayoudh, D. J. McFarland, T. M. Vaughan, and J. R. Wolpaw, "A comparison of classification techniques for the P300 Speller," Journal of Neural Engineering, vol. 3, pp. 299-305, Dec. 2006.

[9] G. Shalk, D.J. McFarland, T. Hinterberger, N. Birbaumer, and J.R. Wolpaw, "BCI2000: A General-Purpose Brain-Computer Interface (BCI) System," IEEE Transactions on Biomedical Engineering, vol. 51, no. 6, May 2004.

[10] L. Montesano, J. Minguez, M. Diaz, and S. Bhaskar, "Towards an Intelligent Wheelchair System for Cerebral Palsy Subjects," IEEE Transactions on Neural Systems and Rehabilitation Engineering, 2009.

[11] L.A. Farwell and E. Donchin, "Talking off the top of your head: toward a mental prosthesis utilizing eve brain potentials," EEG Clinical Neurophysiology, vol. 70, no. 6, pp. 510-23, 1988.

[12] L. Montesano, J. Minguez, and L. Montano, "Lessons learned in integration for sensor-based robot navigation systems," International Journal of Advanced Robotic Systems, vol. 3, no. 1, pp. 85-91, 2006.

[13] L Montesano, J. Minguez, and L.Montano, "Modeling dynamic scenarios for local sensor-based motion planning," Autonomous Robots, 2008 .

[14] A. Ranganathan and S. Koenig, "A reactive architecture with planning on demand," in International Conference on Robotics and Automation, Las Vegas, Nevada, 2003, p. 14621468.

[15] J. Minguez and L. Montano, "Nearness Diagram (ND) Navigation: Collision Avoidance in Troublesome Scenarios," IEEE Transactions on Robotics and Automation, vol. 20, no. 1, pp. 45-59, 2004.

[16] I. Iturrate, J. Antelis, J. Minguez, and A. Kuebler, "Non-Invasive Brain-Actuated Wheelchair based on a P300 Neurophysiological Protocol and Automated Navigation," IEEE Transactions on Robotics, 2009.

[17] S. Mason, J. Kronegg, J. Huggins, M. Fatourechi, and A. Schlogl, "Evaluating the Performance of Self-Paced Brain-Computer Interface Technology," Tech. Rep., Neil Squire Society, Vancouver, Canada, 2006. 